\title{
High Responsivity in GaN Ultraviolet Photodetector Grown on a Periodic Trapezoid-Column Patterned Sapphire Substrate
}

\author{
Kuan-Ting Liu, ${ }^{*}$ Shoou-Jinn Chang, ${ }^{1}$ and Sean $\mathrm{Wu}^{2}$ \\ Department of Electronic Engineering, Cheng Shiu University, \\ No. 840, Chengcing Road, Niaosong District, Kaohsiung City 83347, Taiwan \\ ${ }^{1}$ Institute of Microelectronics, Department of Electrical Engineering Center for Micro/Nano Science and Technology, \\ Advanced Optoelectronic Technology Center, National Cheng Kung University, \\ No. 1, University Road, Tainan City 701, Taiwan \\ ${ }^{2}$ Department of Electronics Engineering and Computer Sciences, Tung Fang Design Institute, \\ No. 110, Dongfang Road, Hunei District, Kaohsiung City 82941, Taiwan
}

(Received August 30, 2016; accepted January 24, 2017)

Keywords: GaN ultraviolet photodetector, metal-semiconductor-metal, periodic trapezoid column-shaped patterned sapphire substrate, responsivity, UV-to-visible rejection ratio

A GaN ultraviolet (UV) photodetector with a metal-semiconductor-metal structure is grown on a periodic trapezoid-column patterned sapphire substrate by metalorganic chemical vapor deposition. Under $5 \mathrm{~V}$ reverse bias, the photodetector fabricated on such a patterned sapphire substrate exhibits a lower dark current, a higher photocurrent, a much larger UV-to-visible rejection ratio, and a $476 \%$ enhancement in the maximum responsivity than those of the photodetector fabricated on a conventional flat sapphire substrate. These phenomena may all be attributed to the reduction in threading dislocation density and the greater number of photogenerated carriers caused by the improved quality of the GaN film, as well as the reflection and/or scattering of unabsorbed photons on the interface between the GaN film and the periodic trapezoid-column pattern of the substrate.

\section{Introduction}

Nitride-based compounds are generally useful for optical devices such as light-emitting diodes (LEDs), laser diodes, and photodetectors (PDs) in green, blue, and ultraviolet (UV) wavelength regions. ${ }^{(1-3)}$ The high-power UV GaN LEDs, for instance, have been intensively studied because they can be used as a pumping source for developing white-light LEDs. White-light LEDs are the most promising solid-state lighting devices to replace conventional incandescent and fluorescent lamps. ${ }^{(4,5)}$ However, the high density of threading dislocations (TDs) in GaN films influences the device characteristics, such as device lifetime and electron mobility. In general, GaN films are usually grown on a sapphire substrate since no suitable substrates can be utilized for GaN-based LEDs or PDs. Owing to the large differences in their lattice constants and thermal expansion coefficients, a large number of TDs arise in the GaN films. ${ }^{(6,7)}$ This can cause a significant degradation in the performance of GaN-based devices. ${ }^{(8,9)}$ Therefore, further reducing the TD density is an important issue for fabricating high-performance UV LEDs or UV PDs. Many

${ }^{*}$ Corresponding author: e-mail: liu@gcloud.csu.edu.tw

http://dx.doi.org/10.18494/SAM.2017.1517 
different growth methods have been applied to the nitrides in order to minimize TDs. ${ }^{(10,11)}$ Epitaxial lateral overgrowth (ELOG) is a commonly used technique to reduce TD density in a GaN film prepared on a sapphire substrate. In this technique, a $\mathrm{SiN}_{x}$ or $\mathrm{SiO}_{2}$ strip mask is grown on GaN templates. ${ }^{(12,13)}$ Lateral overgrowth can then occur through the window regions of the mask. However, ELOG is complex and could result in a low production yield. In recent years, it has been reported that the TD density can be reduced by growing a GaN film on a patterned sapphire substrate (PSS). ${ }^{(14,15)}$ Unlike those in nitride LEDs with a conventional sapphire substrate, the nitride LEDs fabricated on a lamp-form PSS showed improved output power, external quantum efficiency, and reverse leakage current, as reported by Wuu et al. ${ }^{(16)}$ They attributed the improved results to the elimination of TDs and the enhancement of the extraction efficiency using the PSS.

To explore fully the potential functions and development of III-nitrides, a similar concept is theoretically applicable to other GaN-based optoelectronics devices. UV PDs are important devices that can be used in various commercial and military applications, such as space communications, ozone layer monitoring, and flame detection. Various types of GaN-based UV PDs on sapphire substrates, including Schottky diodes, ${ }^{(17)}$ heterojunctions, ${ }^{(18)} \mathrm{p}$-n junctions, ${ }^{(19)} \mathrm{p}-\mathrm{i}-\mathrm{n},{ }^{(20)}$ and metalsemiconductor-metal (MSM) structures, ${ }^{(21)}$ have been reported. Among these structures, MSM PDs have attracted much attention because of their simplicity and high responsivity. MSM structures are also useful in optoelectronic integrated circuits (OEICs) owing to their ease of integration, potential for high speed, and compatibility with field-effect transistor (FET) process technologies. ${ }^{(22)}$ It has been reported that the quality of a GaN Schottky barrier PD grown on a PSS with parallel stripes along the sapphire $<1 \overline{1} 00>$ direction is higher than that prepared on a conventional flat sapphire substrate. $^{(23)}$ The improvement is attributed to the reduction in TD density because of the PSS structure. Moreover, there has been some research on the effects of different patterns of the PSS on the dark current, UV-to-visible rejection ratio, and responsivity of GaN-based p-i-n UV PDs. ${ }^{(24,25)}$ In contrast, only a few studies on GaN-based MSM UV PDs fabricated using the PSS have so far been carried out. Chang et al. found that the GaN-based MSM UV PDs prepared on a PSS can contribute to a much smaller dark leakage current and internal gain than those achieved from the PD fabricated on the conventional flat sapphire substrate. ${ }^{(26)}$ The structure used on the substrate pattern of GaN-based MSM UV PDs in their study is parallel stripes. The improvement of the electrical and optical properties of GaN-based UV LEDs fabricated on a PSS depends on different pattern shapes. ${ }^{(27-29)}$ Consequently, the GaN-based MSM UV PDs may also exhibit a similar behavior to those of GaN-based UV LEDs grown on substrates with different patterns.

The geometrical size of the sapphire patterns is of the micrometer scale. For the same area of the sapphire substrate, a reduced geometrical size of sapphire patterns can increase the number of patterns, and thus increase the opportunity for light scattering from the PSS. ${ }^{(30,31)}$ In addition, a geometrical pattern of a suitable shape and sidewall angle on the sapphire substrate can also increase the reflection of unabsorbed photons resulting in more photogenerated carriers as embedded reflectors, in accordance with Snell's law.

Despite recent advances on patterned substrate in our knowledge, the GaN MSM UV PD grown on a periodic geometrical PSS has not yet been reported. In this study, we investigate, for the first time, the characteristics of a GaN UV PD with an MSM structure fabricated on a periodic trapezoidcolumn patterned sapphire substrate (PTCPSS) by metalorganic chemical vapor deposition (MOCVD). We also report the fabrication and characterization of the GaN MSM UV PD with the PTCPSS. A more detailed study on the structural, electrical, optical, and responsive properties of the PD prepared with such a patterned substrate will be discussed. 


\section{Experimental Procedure}

The PTCPSS used in this study is fabricated by standard photolithography and subsequent inductive couple plasma (ICP) etching in which $\mathrm{Cl}_{2} / \mathrm{BCl}_{3}$ gases are used. For fabricating PTCPSS, a photoresist layer is deposited by a spin coater onto the sapphire substrate as the mask. The photoresist layer is prepared in a periodic column pattern, where the depth and diameter of each column are 2 and $2.8 \mu \mathrm{m}$, respectively. The column array with a spacing of $3.5 \mu \mathrm{m}$ is formed by a standard photolithography process. Next, the sapphire substrate is etched using $\mathrm{Cl}_{2} / \mathrm{BCl}_{3}$ gases in an ICP etcher at $100 \mathrm{~V}$ dc bias. Finally, the photoresist mask is removed using a lift-off solution to complete the PTCPSS structures. The micrograph of the PTCPSS is examined by scanning electron microscopy (SEM), as shown in Fig. 1. Figure 1(a) shows the perspective view of the PTCPSS. By microscopy, it can be observed that the PTCPSS has a pattern of periodic trapezoid columns with a depth of $1.6 \mu \mathrm{m}$ and a sidewall angle of $70^{\circ}$. The top and bottom diameters of the trapezoid columns are 2.8 and $4.6 \mu \mathrm{m}$, respectively, and the trapezoid columns are separated from each other by $1.4 \mu \mathrm{m}$. The top view of the PTCPSS is shown in Fig. 1(b); we can also confirm that the trapezoid-column pattern is uniformly arranged and all the geometrical structures are of equal size.

Afterward, a GaN film is grown on the fabricated PTCPSS by MOCVD. Trimethylgallium (TMGa) and ammonia $\left(\mathrm{NH}_{3}\right)$ are used as the sources of gallium and nitrogen, respectively. The substrate is initially heated to $1100{ }^{\circ} \mathrm{C}$ in hydrogen $\left(\mathrm{H}_{2}\right)$ ambient to remove surface contamination, followed by the lowering of the temperature to $550{ }^{\circ} \mathrm{C}$ to grow a $30-\mathrm{nm}$ thick low-temperature $\mathrm{GaN}$ buffer layer. The temperature is subsequently increased to 1100 ${ }^{\circ} \mathrm{C}$ to grow a $2-\mu \mathrm{m}$-thick undoped $\mathrm{GaN}$ film.

Prior to the fabrication of the MSM PD, the GaN film is dipped in diluted (1:10 of $41 \%)$ hydrofluoric acid and hydrochloric acid water solutions for 1 and $3 \mathrm{~min}$ to remove the native oxide, respectively. $\mathrm{Ni}(5 \mathrm{~nm})$ and $\mathrm{Au}(5 \mathrm{~nm})$ are subsequently deposited onto the GaN film using an electron-beam evaporator as the Schottky contact electrodes for the fabrication of the MSM PD. The MSM PD consists of two interdigitated contact electrodes fabricated by standard photolithography and etching. The fingers of the contact electrodes are $10 \mu \mathrm{m}$ wide and $150 \mu \mathrm{m}$ long with a spacing of $10 \mu \mathrm{m}$, and the detection area is $150 \times 510 \mu \mathrm{m}^{2}$. After contact electrode deposition, a Au layer is

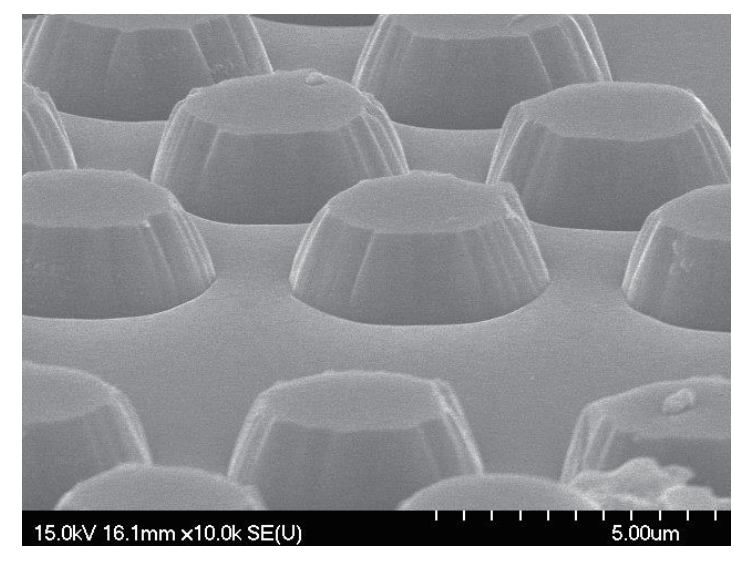

(a)

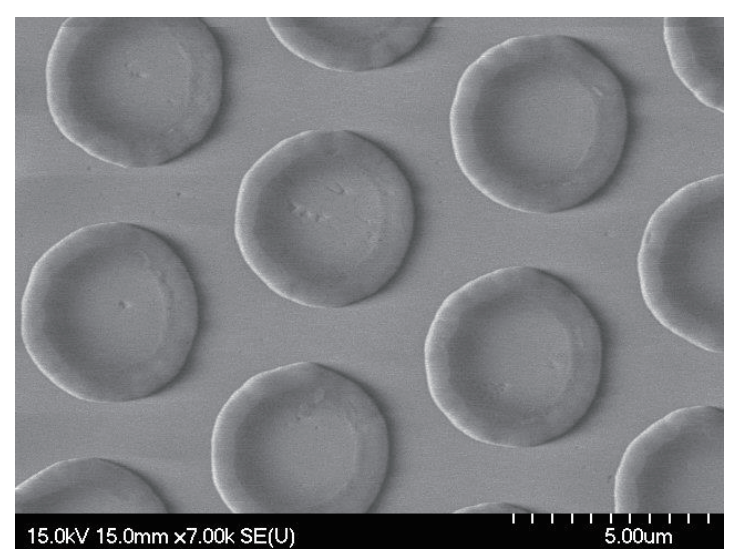

(b)

Fig. 1. SEM images of (a) perspective view and (b) top view of PTCPSS. 
deposited to serve as bonding pads. For comparison, the GaN film and MSM PD on a conventional flat sapphire substrate (CFSS) under the same conditions are also prepared. Note that the fabricated PDs are not packaged and the performances of the PDs are all measured using a probe. The TDs of GaN samples are examined by etch pit density (EPD) measurement. The as-grown GaN samples are characterized by X-ray diffraction (XRD). Photoluminescence (PL) characteristics are determined using a $325 \mathrm{~nm} \mathrm{He-Cd}$ laser as the excitation power, and the carrier concentration is evaluated by Hall effect measurement. A HP-4155B semiconductor parameter analyzer is used to measure the current-voltage $(I-V)$ characteristics of the fabricated PDs in the dark and under illumination. For photocurrent measurements, a deuterium lamp illuminating the front side of the fabricated PDs is used as the light source. A xenon arc lamp is used as the light source for spectral responsivity measurements, and a standard synchronous detection scheme is employed to measure the front-side illuminated detector signal. A calibrated UV-enhanced Si detector is used for signal detection.

\section{Results and Discussion}

For EPD measurements, the $\mathrm{GaN}$ samples are immersed in $\mathrm{H}_{3} \mathrm{PO}_{4}$ at $160{ }^{\circ} \mathrm{C}$ for 3 min. Figure 2 shows the cross-sectional SEM images of the etched GaN samples. In Fig. 2(a), it is found that vertical striations are clearly observed from the GaN film grown on the CFSS. On the other hand, the vertical striations disappeared for the GaN film grown on the PTCPSS, as shown in Fig. 2(b). This observation can be attributed to the enhancement of the lateral overgrowth of the GaN film using the appropriate PTCPSS, which may result in TD reduction in the GaN film.

Figure 3 shows the SEM surface morphologies of the etched GaN films grown on the CFSS and PTCPSS. The EPD of the GaN film grown on the CFSS is estimated to be $1 \times 10^{9}$ $\mathrm{cm}^{-2}$. On the other hand, the EPD is only $3 \times 10^{8} \mathrm{~cm}^{-2}$ for the GaN film grown on the PTCPSS observed from Fig. 3(b). The much lower EPD observed from the GaN film implies that we can effectively reduce the TD density by the PTCPSS. Figure 4 shows the XRD rocking curves of the GaN films grown on the CFSS and PTCPSS. From XRD spectra, the full width at half-maximum (FWHM) of the GaN (0002) peak are 414 and $316 \mathrm{~s}$ for GaN films grown on the CFSS and the PTCPSS, respectively. The sharp and narrow XRD rocking curve for GaN grown on the PTCPSS

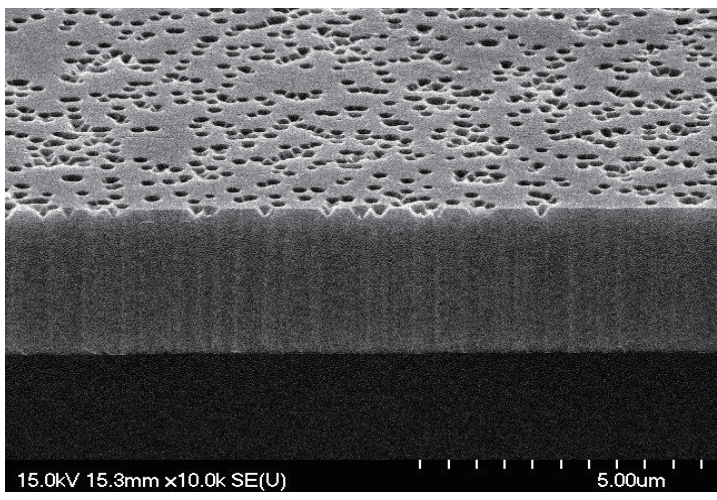

(a)

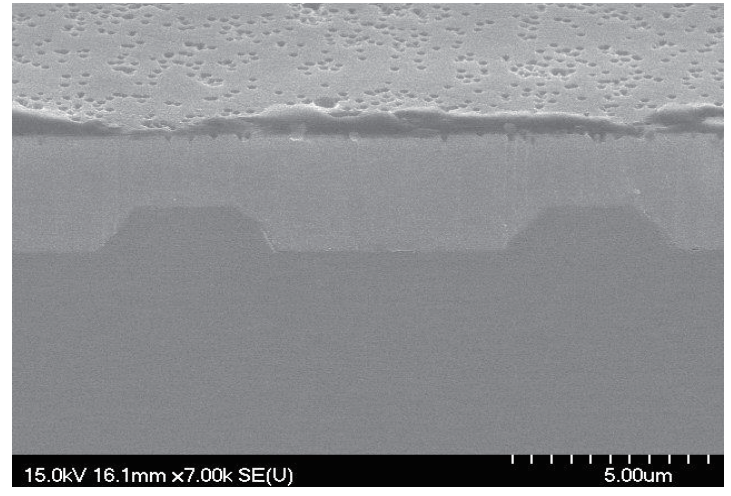

(b)

Fig. 2. Cross-sectional SEM images of etched GaN films grown on (a) CFSS and (b) PTCPSS. 


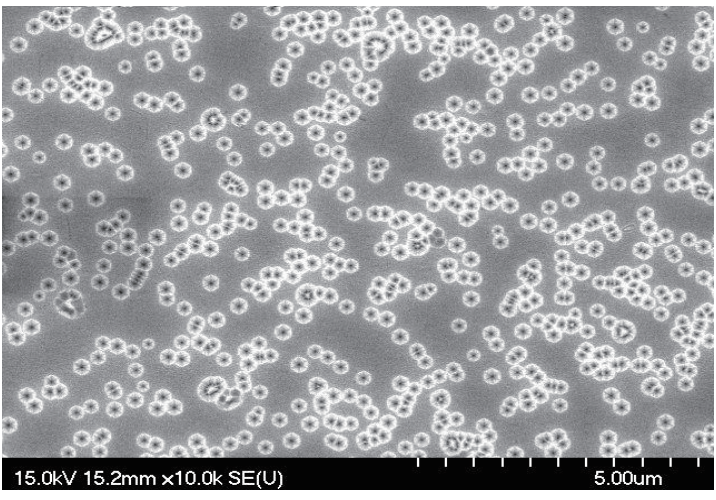

(a)

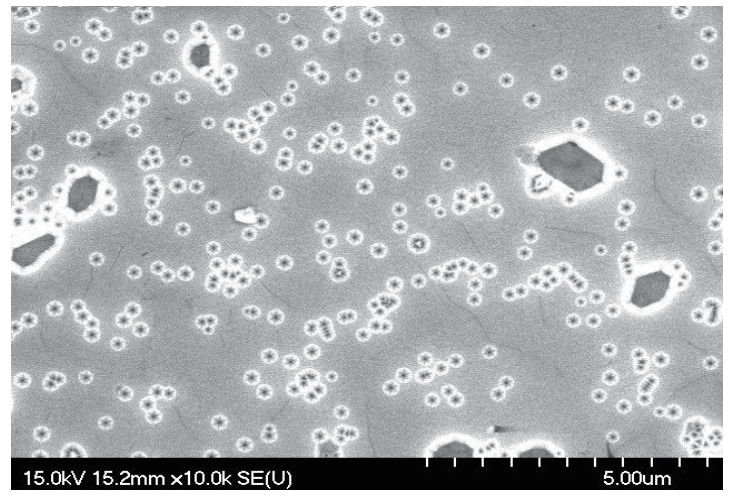

(b)

Fig. 3. SEM surface morphologies of etched GaN films grown on (a) CFSS and (b) PTCPSS.

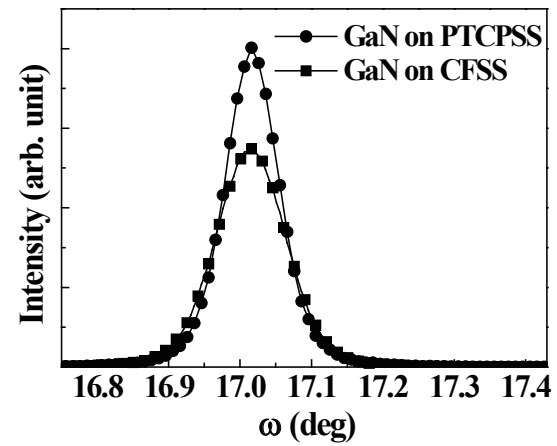

Fig. 4. XRD rocking curves of GaN films grown on CFSS and PTCPSS.

reveals that the crystal quality of the GaN film is markedly improved by using the PTCPSS. Figure 5 shows the PL spectra measured at $20 \mathrm{~K}$ from the GaN films grown on the CFSS and PTCPSS. It can be seen clearly that the PL spectrum of the GaN film grown on the CFSS exhibits a relatively weak band-edge emission at $3.468 \mathrm{eV}$ and a notorious yellow band (YB) emission near $2.3 \mathrm{eV}$. ${ }^{(32)}$ In contrast, a strong band-edge-emission PL peak with a sharp 14 meV FWHM is observed from the GaN film grown on the PTCPSS. The increase in the PL intensity and the absence of YB emission indicate that the crystal quality is improved and the TD density is reduced for the GaN film grown on the PTCPSS. From Hall effect measurement, the residual carrier concentrations of the GaN film are found to be $1.37 \times 10^{17}$ and $3.22 \times 10^{16} \mathrm{~cm}^{-3}$, while the carrier mobility is 160 and $210 \mathrm{~cm}^{2} / \mathrm{Vs}$ at room temperature for the CFSS and the PTCPSS, respectively. The reduction in the residual carrier concentration demonstrates again that the crystal quality is improved for the GaN film grown on the PTCPSS. The results of Hall effect measurement are in reasonable agreement with the results of XRD and PL measurements.

Figure 6 shows the $I-V$ characteristics of the two fabricated MSM PDs measured in the dark and under illumination. With $5 \mathrm{~V}$ reverse bias, the dark leakage currents of $5.3 \times 10^{-14}$ and $1.2 \times 10^{-11}$ A are observed for the PDs fabricated on the PTCPSS and the CFSS, respectively, corresponding to a reduction of three orders of magnitude. It is also found that the dark leakage current of our PD with the PTCPSS is $5.3 \times 10^{-14} \mathrm{~A}$, whereas that reported in Ref. 26 is approximately $7 \times 10^{-12}$ A. The structure used on the substrate pattern of GaN MSM UV PDs in Ref. 26 is parallel stripes. 


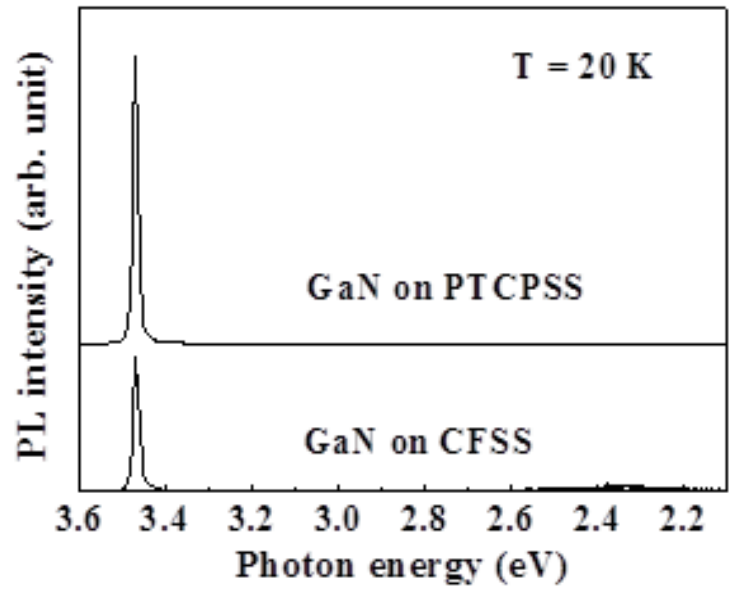

Fig. 5. PL spectra measured at $20 \mathrm{~K}$ from GaN films grown on CFSS and PTCPSS.

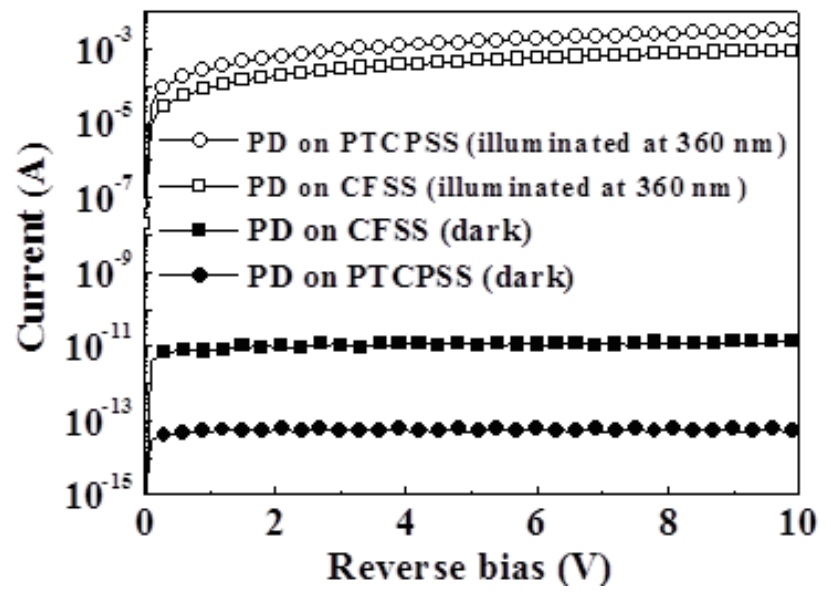

Fig. 6. $I-V$ characteristics of PDs fabricated on CFSS and PTCPSS measured in the dark and under illumination.

The much smaller dark leakage current for our PD may be attributed to the effective suppression of TD density because of the different substrate pattern. ${ }^{(33)}$ The reduced TD density of the PD with the PTCPSS leads to a smaller probability of the occurrence of leakage current paths. On the other hand, it is found that the photocurrent from the PD with the PTCPSS is higher than that from the PD with the CFSS by about one order of magnitude. Under $5 \mathrm{~V}$ reverse bias and $360 \mathrm{~nm}$ wavelength light illumination, the photocurrents of the PDs are $1.6 \times 10^{-3}$ and $4.6 \times 10^{-4} \mathrm{~A}$ with the PTCPSS and the CFSS, respectively. To clarify the effect of the substrate on the PD photocurrent, the transmittance spectra of the GaN films grown on the CFSS and PTCPSS are determined using a UV-Visible (UV-VIS) spectrophotometer, as shown in Fig. 7. The oscillations of transmittance spectra from both GaN samples are due to interference behavior. The observation of a steeper transition edge at about $360 \mathrm{~nm}$ (i.e., $3.4 \mathrm{eV}$ ) from the $\mathrm{GaN}$ film grown on the PTCPSS reflects the bandgap of the GaN and indicates that the crystal quality is good owing to the reduction of TD density. When the incident photons are absorbed by the high-quality GaN film, what generates carriers and then contributes to the large photocurrent. The partially unabsorbed photons that reach the substrate are reflected and/or scattered back to the GaN film via a suitably geometrical substrate, which probably generates carriers again and subsequently offers the photocurrent. Therefore, the fabricated periodic trapezoid-column pattern may improve the reflection and/or scattering of unabsorbed photons on the interface between the GaN film and the PTCPSS. Compared with the PD with the CFSS, the higher photocurrent generated from the PD with the PTCPSS may result from the large number of photogenerated carriers generated by the improved quality of the GaN film and the reflection and/or scattering of unabsorbed photons on the periodic trapezoid-column pattern of the substrate. As a result, the photocurrent-to-dark current contrast is higher than ten orders of magnitude for the PD fabricated on the PTCPSS with a $5 \mathrm{~V}$ bias voltage; this current contrast is higher than that of the PD fabricated on the CFSS.

Figure 8 shows the spectral responses of both MSM PDs biased at $5 \mathrm{~V}$. It is found that the cutoff wavelength occurs at $360 \mathrm{~nm}$ for both PDs. The maximum responsivities at $360 \mathrm{~nm}$ are 0.98 and $0.17 \mathrm{~A} / \mathrm{W}$ for the PDs fabricated on the PTCPSS and the CFSS, respectively. The maximum responsivity of $0.98 \mathrm{~A} / \mathrm{W}$ for our PD is also larger than that of around $0.38 \mathrm{~A} / \mathrm{W}$ reported in Ref. 26. In addition, we can also observe the responsivity drop in a sharp cut-off region of $10 \mathrm{~nm}$ for 


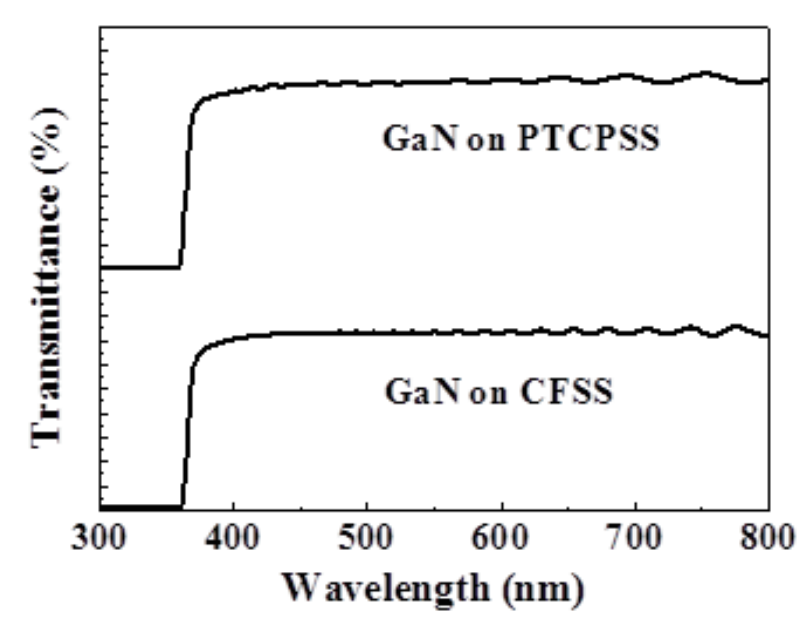

Fig. 7. Transmittance spectra of GaN films grown on CFSS and PTCPSS.

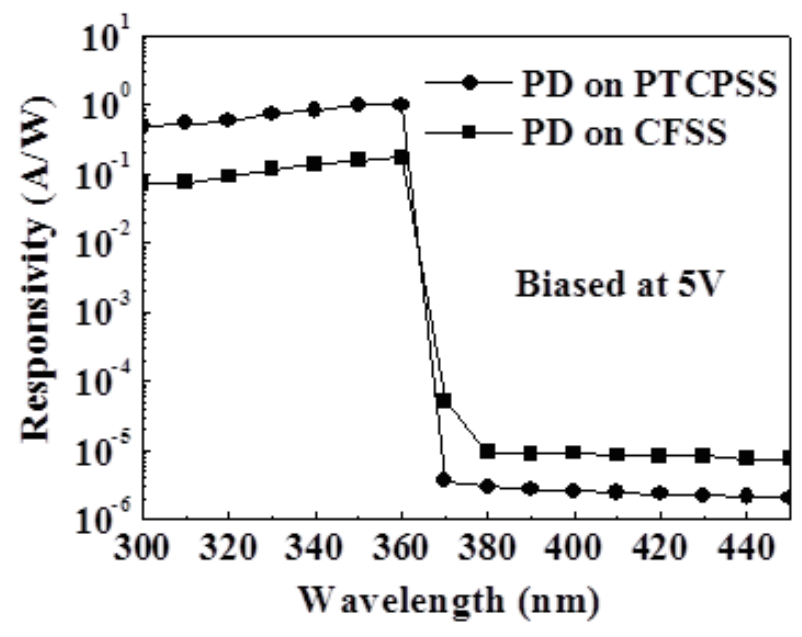

Fig. 8. Spectral responses of PDs fabricated on CFSS and PTCPSS at $5 \mathrm{~V}$ bias.

the PD with the PTCPSS. In contrast, the cut-off region of responsivity for the PD with the CFSS is $20 \mathrm{~nm}$. The UV-to-visible rejection ratio can be defined as the responsivity measured at $360 \mathrm{~nm}$ divided by the responsivity measured at $450 \mathrm{~nm}$. With such a definition, the UV-to-visible rejection ratios are evaluated to be $4.8 \times 10^{5}$ and $2.3 \times 10^{4}$ for PDs fabricated on the PTCPSS and the CFSS, respectively. It is found that a much higher the maximum responsivity can be achieved, the PD response drops in a small cut-off region, and the UV-to-visible rejection ratio is larger for the PD fabricated on the PTCPSS. These observations may be attributed to the reduction in TD density and the improved quality of the GaN film, as well as the reflection and/or scattering of unabsorbed photons on the periodic trapezoid-column pattern of the substrate. In other words, the experimental results demonstrate that a high-performance MSM UV PD based on GaN can be achieved by using a PTCPSS with a suitable geometrical pattern.

\section{Conclusions}

The GaN UV PD with the MSM structure fabricated on the PTCPSS by MOCVD was systematically investigated. Compared with the PD with the CFSS, we achieved a lower dark current of $5.3 \times 10^{-14} \mathrm{~A}$, a higher photocurrent of $1.6 \times 10^{-3} \mathrm{~A}$, and a $476 \%$ enhancement in the maximum responsivity from the PD with the PTCPSS at $5 \mathrm{~V}$ reverse bias. It was also found that the PD response drops in a small cut-off region of $10 \mathrm{~nm}$ for the PD with the PTCPSS under $5 \mathrm{~V}$ applied bias. Furthermore, we also observed that the UV-to-visible rejection ratio of the PD with the PTCPSS was $4.8 \times 10^{5}$, while that of the PD with the CFSS was only about $2.3 \times 10^{4}$ under 5 $\mathrm{V}$ applied bias. These improved results observed from the PD fabricated on the PTCPSS may be attributed to the reduction in the TD density and the improved quality of the GaN film, as well as the reflection and/or scattering of unabsorbed photons by the PTCPSS with a suitable geometrical pattern.

In addition, the experimental results also showed that the dark leakage current and maximum responsivity of our GaN MSM UV PD with a suitably geometrically patterned substrate are better than those reported in Ref. 26. The structure used in the substrate pattern of GaN MSM UV PDs in Ref. 26 was parallel stripes. Using the PTCPSS, the TD density may be effectively suppressed by choosing an appropriate substrate pattern. 


\section{References}

1 S. Nakamura, M. Senoh, N. Iwasa, and S. Nagahama: Jpn. J. Appl. Phys. 34 (1995) L797.

2 S. Nakamura, M. Senoh, S. Nagahama, N. Iwasa, T. Yamada, T. Matsushita, Y. Sugimoto, and H. Kiyoku: Jpn. J. Appl. Phys. 36 (1997) L1059.

3 C. K. Wang, S. J. Chang, Y. K. Su, C. S. Chang, Y. Z. Chiou, C. H. Kuo, T. K. Lin, and J. J. Tang: Mater. Sci. Eng. B 112 (2004) 25.

4 B. Damilano, N. Grandjean, C. Pernot, and J. Massies: Jpn. J. Appl. Phys. 40 (2001) L918.

5 I. Ozden, E. Makarona, A. V. Nurmikko, T. Takeuchi, and M. Krames: Appl. Phys. Lett. 79 (2001) 2532.

6 M. L. Tu, Y. K. Su, S. J. Chang, and R. Chuang: J. Cryst. Growth 298 (2007) 744.

7 I. Akasaki: J. Cryst. Growth 198/199 (1999) 885.

8 S. D. Lester, F. A. Ponce, M. G. Craford, and D. A. Steigerwald: Appl. Phys. Lett. 66 (1995) 1249.

9 M. Iwaya, T. Takeuchi, S. Yamaguchi, C. Wetzel, H. Amano, and I. Akasaki: Jpn. J. Appl. Phys. 37 (1998) L316.

10 X. Li, S. G. Bishop, and J. J. Coleman: Appl. Phys. Lett. 73 (1998) 1179.

11 I. Kidoguchi, A. Ishibashi, G. Sugahara, and Y. Ban: Appl. Phys. Lett. 76 (2000) 3768.

12 P. Fini, L. Zhao, B. Moran, M. Hansen, H. Marchand, J. P. Ibbetson, S. P. Denbaars, U. K. Mishra, and J. S. Speck: Appl. Phys. Lett. 75 (1999) 1706.

13 S. Takumi, S. Hiroki, Y. Katsunori, Y. Masahito, and H. Kazumasa: J. Cryst. Growth 189 (1998) 67.

14 D. S. Wuu, W. K. Wang, K. S. Wen, S. C. Huang, S. H. Lin, S. Y. Huang, and C. F. Lin: Appl. Phys. Lett. 89 (2006) 161105.

15 T. V. Cuong, H. S. Cheong, H. G. Kim, H. Y. Kim, C. H. Hong, E. K. Suh, H. K. Cho, and B. H. Kong: Appl. Phys. Lett. 90 (2007) 131107.

16 D. S. Wuu, W. K. Wang, W. C. Shih, R. H. Horng, C. E. Lee, W. Y. Lin, and J. S. Fang: IEEE Photon. Technol. Lett. 17 (2005) 288.

17 Q. Chen, J. W. Yang, A. Osinsky, S. Gangopadhyay, B. Lim, M. Z. Anwar, M. Asif Khan, D. Kuksenkov, and H. Temkin: Appl. Phys. Lett. 70 (1997) 2277.

18 Z. C. Huang, J. C. Chen, and D. Wickenden: J. Cryst. Growth 170 (1997) 362.

19 E. Monroy, E. Muñoz, F. J. Sánchez, F. Calle, E. Calleja, B. Beaumout, P. Gibart, J. A. Muñoz, and F. Cussó: Semicond. Sci. Technol. 13 (1998) 1042.

20 E. Monroy, M. Hamilton, D. Walker, P. Kung, G. J. Sánchez, and M. Razeghi: Appl. Phys. Lett. 74 (1999) 1171.

21 Z. M. Zhao, R. L. Jiang, P. Chen, D. J. Xi, Z. Y. Luo, R. Zhang, B. Shen, Z. Z. Chen, and Y. D. Zheng: Appl. Phys. Lett. 77 (2000) 444.

22 D. G. Parker and P. G. Say: Electron. Lett. 22 (1986) 1266.

23 S. J. Chang, S. M. Wang, T. P. Chen, S. J. Young, Y. C. Lin, S. L. Wu, and B. R. Huang: J. Electrochem. Soc. 157 (2010) J212.

24 G. Wang, H. Lu, D. Chen, F. Ren, R. Zhang, and Y. Zheng: IEEE Photon. Technol. Lett. 25 (2013) 652.

25 H. Huang, D. Yan, G. Wang, F. Xie, G. Yang, S. Xiao, and X. Gu: Chin. Opt. Lett. 12 (2014) 092301.

26 S. J. Chang, Y. D. Jhou, Y. C. Lin, S. L. Wu, C. H. Chen, T. C. Wen, and L. W. Wu: IEEE Photon. Technol. Lett. 20 (2008) 1866.

27 J. H. Lee, J. T. Oh, J. S. Park, J. W. Kim, Y. C. Kim, J. W. Lee, and H. K. Cho: Phys. Status Solidi C 3 (2006) 2169.

28 T. X. Lee, K. F. Gao, W. T. Chien, and C. C. Sun: Opt. Express 15 (2007) 6670.

29 J. J. Chen, Y. K. Su, C. L. Lin, S. M. Chen, W. L. Li, and C. C. Kao: IEEE Photon. Technol. Lett. 20 (2008) 1193.

30 C. C. Wang, H. Ku, C. C. Liu, K. K. Chong, C. I. Hung, Y. H. Wang, and M. P. Houng: Appl. Phys. Lett. 91 (2007) 121109.

31 H. Gao, F. Yan, Y. Zhang, J. Li, Y. Zeng, and G. Wang: J. Appl. Phys. 103 (2008) 014314.

32 D. J. Dewsnip, A. V. Andrianov, I. Harrison, J. W. Orton, D. E. Lacklison, G. B. Ren, S. E. Hooper, T. S. Cheng, and C. T. Foxon: Semicond. Sci. Technol. 13 (1998) 500.

33 S. W. Lee, D. C. Oh, H. Goto, J. S. Ha, H. J. Lee, T. Hanada, M. W. Cho, T. Yao, S. K. Hong, H. Y. Lee, S. R. Cho, J. W. Choi, J. H. Choi, J. H. Jang, J. E. Shin, and J. S. Lee: Appl. Phys. Lett. 89 (2006) 132117. 